\title{
Breast Density and Breast Cancer
}

\author{
PAMELA HALLQUIST VIALE, RN, MS, CNS, ANP
}

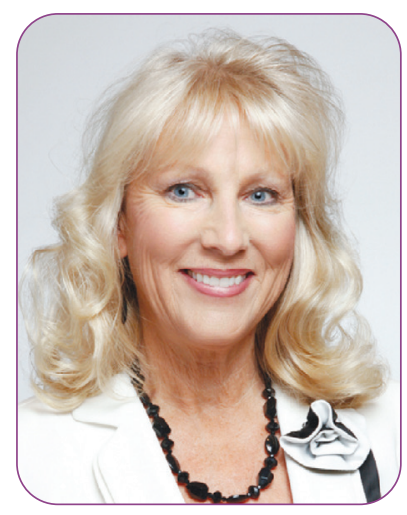

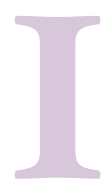

f a woman receives a screening mammogram that demonstrates high breast density, it is mandated that the mammogram report include a summary about how dense breast tissue is a common occurrence and that the density can make it harder to evaluate the results of the mammogram exam (American Cancer Society, 2019). Information about breast density is important, because women with higher breast density have a higher risk of breast cancer than women with less dense breast tissue (American Cancer Society, 2019).

As a woman with fairly dense breasts, I've received a mammogram report with a summary similar to this every time I've had an exam. However, once a patient is informed of her results on mammogram, there are no clearly established guidelines for additional testing that demonstrate value in studies. It is frustrating to receive such a report without additional information to guide the patient on appropriate next steps.

\section{BREAST DENSITY CATEGORIES AND THE DENSE STUDY}

There are four categories of breast density, ranging from almost all fatty tissue to extremely dense tissue with very little fat. With higher breast density, the radiologist has a more difficult time identifying cancerous tumors in the breast tissue. Studies have shown that additional imaging can improve the identification of some breast cancers not seen on mammograms, such as breast ultrasound and MRI exams (American Cancer Society, 2019). However, although MRI exams are more sensitive, mammography is actually a more specific exam (Longo, 2019).

A recently published study in the New England Journal of Medicine by authors Bakker and colleagues (2019), the Dense Tissue and Early Breast Neoplasm Screening clinical trial, or DENSE, discusses the results of a randomized clinical trial from the Netherlands examining women with extremely dense breast tissue receiving screening. Twenty percent (8,061 of 40,373 women) of the participants were randomly assigned to be invited for additional MRI screening after mammography; out of this initial group, 59\% agreed to participate. The primary outcome of the study was the between-group difference in the incidence of interval cancers detected during a 2-year screening period.

\section{Results}

The women in the intent-to-screen population in the MRI-invitation group (including those participants receiving MRI and those who de- 
clined) demonstrated an interval cancer rate of 2.5 per 1,000 screenings vs. 5.0 cancers per 1,000 screenings for the mammography-only group (Bakker et al., 2019). The women who received supplemental MRI had a cancer-detection rate of 16.5 per 1,000 screenings. The positive predictive value was $17.4 \%$ ( $95 \%$ confidence interval $[\mathrm{CI}]=$ $14.2 \%-21.2 \%)$ for patients recalled for additional testing and $26.3 \%$ (95\% CI $=21.7 \%-31.6 \%)$ for biopsy (Bakker et al., 2019). Almost 10\% of women who received MRI screening exams were recalled for abnormal scans; of the 300 women who received a biopsy, 79 were determined to have breast cancer (Bakker et al., 2019).

\section{Clinical Dilemma}

The results of the DENSE study demonstrate that the risk of interval cancers is reduced by $50 \%$ by adding an MRI exam to a negative mammogram for women with dense breasts (Longo, 2019). The strength of this study's finding remains in its randomized design (Bakker et al., 2019). However, we don't yet have information that will definitively show that MRI screening in this patient population will improve survival; adding regular MRI exams to mammography coupled with additional biopsy exams will also lead to higher costs, false positives, and the potential (although low) for complications.

As a woman with dense breast tissue and a clinician, I find the study results intriguing, yet the need for further data remains. Until we have randomized clinical trials showing survival advantages, should MRI exams following traditional mammography become the gold standard for women with dense breast tissue? The American Society of Breast Surgeons guidelines call for women younger than 50 with a history of breast cancer or those with dense breasts to receive a mammogram every year with supplemental imaging as recommended by their doctors; the ACS guidelines currently state that there is not enough evidence to make a recommendation for yearly MRI screening for extremely dense breast tissue, and if used, should be used in conjunction with yearly mammograms
(ACS, 2019; American Society of Breast Surgeons, 2019). Until we have definitive data results showing survival advantage, patients with dense breasts should be offered the possibility of further testing, taking into account additional information regarding patient risk factors (family and personal history).

\section{IN THIS ISSUE}

Starting us off in the first issue of the year are Dr. Casey Brown and her coauthors detailing the impact of survivorship care plans in patients with glioblastoma at their institution. Our Practice Matters article by Drs. M. Tish Knobf and Sangchoon Jeon outlines a randomized controlled trial studying the impact of a fitness center exercise intervention for breast cancer survivors. Dr. Theresa Wicklin Gillespie shares her expertise on the new oral oncolytics for breast cancer and approaches to enhancing adherence in a CEcertified article. We have two case studies in this issue: one on managing pancreatitis and another on screening for a rare but life-threatening disorder, dihydropyrimidine dehydrogenase deficiency. Get a comprehensive review of the new drug gilteritinib, and test your knowledge on what to do for a patient with gastric cancer with gastric outlet obstruction in our Diagnostic Snapshot.

\section{References}

American Cancer Society. (2019). Breast density and your mammogram report. Retrieved from https://www.cancer.org/cancer/breast-cancer/screening-tests-and-early-detection/mammograms/breast-density-and-yourmammogram-report.html

American Society of Breast Surgeons. (2019). American Society of Breast Surgeons Issues Updated Breast Cancer Screening Guidelines. Retrieved from https://www. breastcancer.org/research-news/asbrs-issues-updatedscreening-guidelines

Bakker, M. F., de Lange, S. V., Pijnappel, R. M., Mann, R. M., Peeters, P. H. M., Monninkhof, E. M.,...van Gils, C. H. (2019). Supplemental MRI screening for women with extremely dense breast tissue. New England Journal of Medicine, 381(22), 2091-2102. https://doi.org/10.1056/ NEJMoa1903986

Longo, D. (2019). Detecting breast cancer in women with dense breasts. New England Journal of Medicine, 381, 22. https://doi.org/10.1056/NEJMe1912943 